\title{
Temperature effect on light polarization in uniaxial crystals
}

\author{
M.R. Kulish ${ }^{1}$, V.M. Litvinova ${ }^{2}$, M.I. Malysh ${ }^{3}$, I.O. Sokolovskyi ${ }^{1}$ \\ ${ }^{I} V$. Lashkaryov Institute of Semiconductor Physics, NAS of Ukraine, \\ 41, prospect Nauky, 03028 Kyiv, Ukraine \\ ${ }^{2}$ Cornell University, Ithaca, NY 14850, U.S.A. \\ ${ }^{3}$ National Transport University, 22 B, of. 351, Novatoriv str., 02090 Kyiv, Ukraine
}

\begin{abstract}
It has been shown that changes in temperature of uniaxial single crystal are accompanied by changes in the polarization plane angular position and the light intensity that subsequently passes through the polarizer, uniaxial crystal, and analyzer. These effects introduce systematic errors in the results of researching the nonlinear processes. To minimize this harmful effect, polarization researches must be carried out at a constant sample temperature. For example, research of the nonlinear polarization processes in $\mathrm{CdS}$ should be done with a sample temperature uncertainty no higher than $\pm 1 \mathrm{~K}$.
\end{abstract}

Keywords: uniaxial crystal, light polarization, temperature dependence, thermal expansion.

Manuscript received 07.10.15; revised version received 12.01.16; accepted for publication 16.03.16; published online 08.04.16.

\section{Introduction}

As usual, nonlinear light absorption of uniaxial crystals is investigated when the plane of polarization is parallel or perpendicular to the optical axis $C$ (see, e.g. [1,2]). However, the number of papers (see [3]) devoted to nonlinear polarization absorption properties is extremely limited. We believe that the dependence of parameters, such as thickness and refractive index, on changing the uniaxial single crystals with temperature is the reason of it. Thus, the change in the state of polarization of light results from not only nonlinear absorption, but also from the changes in temperature of the sample.

In this work, we investigated the influence of temperature inherent to the uniaxial single crystal $\mathrm{CdS}$ on polarization of light propagating in it.

\section{Change in polarization of light during its propagation in uniaxial crystal}

A simplified diagram illustrating propagation of linearly polarized light in a uniaxial crystal is shown in Fig. 1. Let a parallel light beam sequentially propagates through the polarizer, uniaxial crystal, and analyzer. Let the optical axis of the crystal be in the plane that is normal to propagation direction of the light beam. Assume that the optical axis coincides with the ordinate. Let the angle between the electric vector of the electromagnetic wave and the optical axis is equal to $\alpha$ at the input and $\beta$ at its output (Fig. 1). Assume the $x$-axis is orthogonal to the axis of ordinates. Let the directions of vectors OP and $\mathrm{OA}$ correspond to the directions of electromagnetic wave oscillations after passing the polarizer and before 
entering the analyzer, respectively. If the plane of polarization is rotated after passing through the uniaxial crystal, then $\alpha \neq \beta$.

We can assume that, at the uniaxial crystal entrance, the light beam consists of two components that propagate in the same direction. One light vector $\mathbf{E}$ is parallel to the optical axis $C$, and another vector $\mathbf{E}$ is perpendicular to the optical axis. Since the velocity of these components is different, one component lags relatively to another. Consequently, the result is a rotation of light polarization plane at the output from the crystal and, in this case, there is the following dependence of light intensity at the analyzer output [4]:

$I=I_{0} \cos ^{2}(\alpha-\beta)-I_{0} \sin 2 \alpha \sin 2 \beta \sin ^{2}(\delta / 2)$,

where $I_{0}$ is the intensity of light at the entrance polarizer, $I$ - intensity of light at the output from the analyzer, and $\delta$ - phase difference for light components.

$\delta=\frac{2 \pi d\left(n_{e}-n_{o}\right)}{\lambda}$,

where $d$ is the thickness of the uniaxial crystal, $n_{o}$ and $n_{e}$ are the refractive indices of ordinary ray (propagating along the axis $C$ ) and extraordinary ray (propagating orthogonally to the axis $C$ ) respectively, and $\lambda$ is the wavelength.

The temperature dependence of $d$ is given by:

$d=d_{o}(1+\gamma \cdot \Delta T)$

where $\gamma$ is the coefficient of linear expansion, $\Delta T=T-T_{o}, T$ and $d$ are the final temperature and the crystal thickness, respectively, and $T_{0}$ and $d_{0}-$ initial temperature and thickness of the crystal, respectively.

In (2) $n_{o}, n_{e}$, and $d$ are dependent on temperature. The temperature dependence of the sample thickness and the difference between the refractive indices $n_{o}$ and $n_{e}$ are characteristics of a particular semiconductor.

\section{Effect of the temperature CdS single crystal state on light polarization}

The simplified diagram of the analysis of light polarization during distribution in $\mathrm{CdS}$ planar plate is shown in Fig. 1. Helium-neon laser serves as a light source $(\lambda=632.8 \mathrm{~nm})$. The double Fresnel rhombus is a polarizer. The Glan prism is the analyzer. The detector is photodiode FD-24K. The sample temperature was controlled using the copper-constantan thermocouple. The sample temperature was governed by the heating stove.

The data in Fig. 2 show that, when the temperature changes, the polarization plane should rotate. To test this assumption, we measured the temperature dependence of the angle of rotation of the polarization plane. To do this, at a given sample temperature, we determined the maximum light intensity value and measured the angular position of the analyzer. In this case, the polarization plane at the output from the sample coincides with the position of the polarization plane at the output of analyzer.

The experimental results are shown in Fig. 3 (points). To find the functions necessary to approximate this dependence, we equate the derivative $d I / d \beta$ to zero. After some simple transformations, we obtain the following relationship between $\beta$ and $\Delta T$

$$
\operatorname{tg} 2 \dot{\Gamma} \operatorname{Actg} 2 \alpha=\cos \left\{\left[\pi\left(n_{e}-n_{o}\right) / \lambda\right] \cdot\left[d_{o}(1+\gamma \Delta T)\right]\right\} .
$$

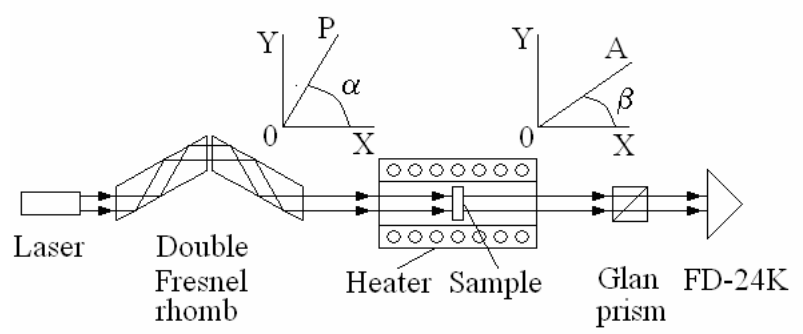

Fig. 1. Simplified scheme for studying the impact of external factors on polarization of light propagating in the uniaxial crystal. $\alpha$ and $\beta$ are the angles between the optical axis and light vector at the input and output of the sample, respectively. FD-24K is a silicon photodiode.

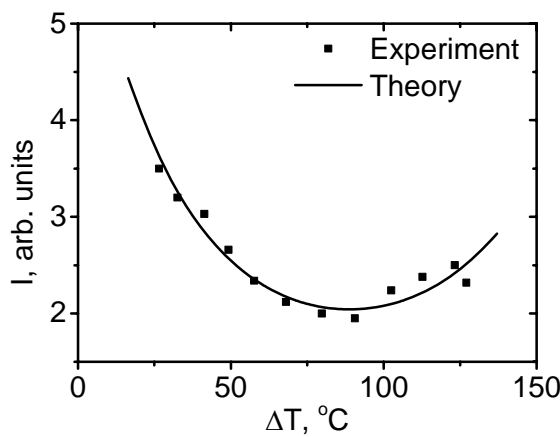

Fig. 2. Dependence of the light intensity outputing from the analyzer versus the sample temperature. Parameters of the plane-parallel CdS single crystal: $d_{0}=2.1 \mathrm{~mm},\left(n_{o}-n_{e}\right)=$ $0.017, \lambda=632.8 \mathrm{~nm}, \gamma=2.5 \cdot 10^{-5} \mathrm{C}^{-1}, \alpha=\beta=70^{\circ}, I_{0}=10 \mathrm{~mW}$. Points show the experimental data, the solid curve was calculated using the formula (1)

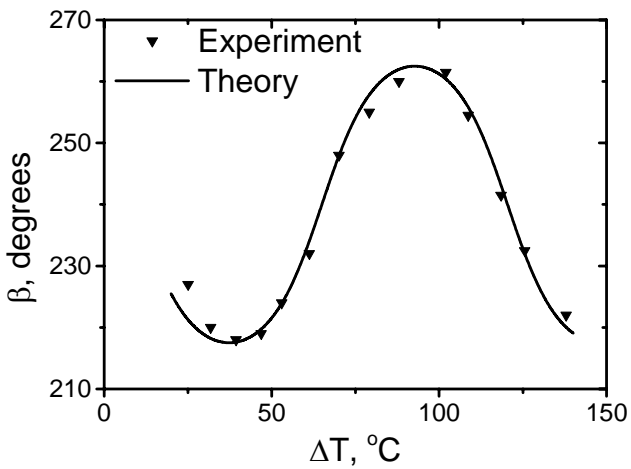

Fig. 3. Temperature dependence of the polarization plane rotation angle for the CdS sample (thickness is equal $0.21 \mathrm{~cm}$ ). Points stand for the experimental data, the solid curve was calculated using the formula (4). 
Calculated using the formula (4), the dependence of the polarization plane rotation angle versus temperature (Fig. 3, solid curve) is in excellent agreement with experimental data (Fig. 3, points).

Usually, nonlinear absorption polarization research is performed at room temperature for a long time, so under conditions of variations in the room temperature the sample temperature must be maintained invariable.

In particular, the data of Figs. 2 and 3 show that during polarization studies of nonlinear processes in $\mathrm{CdS}$, the sample temperature should be kept constant during measurements with an error less than $\pm 1 \mathrm{~K}$.

\section{Conclusion}

When investigating polarization in uniaxial crystals, one need to stabilize the sample temperature to exclude systematic errors related with the temperature dependence of the sample thickness and refractive index difference between respective values for ordinary and extraordinary rays.

\section{References}

1. V.P. Kunets, Polarization self-action effect and absorption saturation in CdSe single crystals // phys. status solidi (b), 209, p. 179-186 (1998).

2. M.R. Kulish, M.I. Malysh, G.L. Isaienko, V.M. Litvinova, Influence of absorption saturation on the shape of CdSe absorption edge // Semiconductor Physics, Quantum Electronics \& Optoelectronics, 17, N 4, p. 349-352 (2014).

3. M.R. Kulish, M.P. Lisitsa, N.I. Malysh, Influence of polarization azimuth on two-photon absorption in CdS // Semiconductor Physics, Quantum Electronics \& Optoelectronics, 8, N 4, p. $72-73$ (2005).

4. R.W. Ditchburn, Light. New York Intersci. Publ., 1964.

5. N.I. Vitrikhovskiy, L.F. Gudymenko, A.F. Maznichenko et al., Dispersion of the refractive indices of $\mathrm{CdS}_{\mathrm{x}} \mathrm{Se}_{1-\mathrm{x}}$ and CdTe single crystals in the visible and infrared spectrum ranges // Ukr. fizychnyi zhurnal, 12(5), p. 796-800 (1967), in Ukrainian.

6. Sadao Adachi, The Handbook on Physical Properties of Semiconductors. Vol. 3: II-VI Compound Semiconductors. Springer US, 2012. 